\title{
The state of complement in COVID-19
}

\author{
Behdad Afzali(i), Marina Noris (iD, Bart N. Lambrecht and Claudia Kemper(D)
}

Abstract | Hyperactivation of the complement and coagulation systems is recognized as part of the clinical syndrome of COVID-19. Here we review systemic complement activation and local complement activation in response to the causative virus severe acute respiratory syndrome coronavirus 2 (SARS-CoV-2) and their currently known relationships to hyperinflammation and thrombosis. We also provide an update on early clinical findings and emerging clinical trial evidence that suggest potential therapeutic benefit of complement inhibition in severe COVID-19.

COVID-19, caused by severe acute respiratory syndrome coronavirus 2 (SARS-CoV-2), is the largest pandemic disease of humans in the past century. Thus far, approximately 225 million cases and more than 4.5 million deaths have been attributed to COVID-19 globally ${ }^{1}$. The clinical manifestations of COVID-19 can be highly variable, ranging from mild upper respiratory tract symptoms in most cases to severe, life-threatening, multi-organ disease ${ }^{2}$. Up to one-third of patients affected by COVID-19 will develop persistent symptoms ${ }^{3}$, and some will have permanent end-organ dysfunction pursuant to tissue fibrosis ${ }^{4}$. Despite the emerging epidemiological data, the underlying pathogenesis of COVID-19 and its optimal treatment are still poorly understood. Undoubtedly, detailed mechanistic understanding of this disease will enable more effective therapies for its treatment, save lives and preserve healthy tissues.

The complement system is an ancient, evolutionarily conserved and non-redundant component of immunity. It is classically viewed as a liver-derived and plasma-operative system constantly scanning the blood and interstitial fluids for invading pathogens and self-derived noxious antigens. Pathogen sensing triggers one or several complement activation pathways (the lectin pathway, the classical pathway or the alternative pathway) and cleavage-mediated activation of the key components $\mathrm{C} 3$ and $\mathrm{C} 5$ by $\mathrm{C} 3$ and $\mathrm{C} 5$ convertases, respectively, into bioactive $\mathrm{C} 3 \mathrm{a}$ and $\mathrm{C} 3 \mathrm{~b}$ and $\mathrm{C} 5 \mathrm{a}$ and C5b. C3a and C5a are anaphylatoxins that mediate the general inflammatory reaction, $\mathrm{C} 3 \mathrm{~b}$ is a major opsonin that induces tagging and phagocytic uptake of pathogens and C5b seeds the formation of the membrane attack complex (MAC; C5b-9), which directly lyses pathogens ${ }^{5}$. Owing to its central role in the detection and removal of pathogens, complement should intuitively be protective during SARS-CoV-2 infection. However, several lines of evidence implicate this system as a key component in the pathogenesis of COVID-19, and emerging trial data suggest clinical benefits from targeting this system therapeutically (FIG. 1). Thus, complement, like many components of the immune system, could be viewed as a 'double-edged sword', with its dysregulation leading to harmful effects. Of course, how and why the normally protective complement system becomes pathogenic in COVID-19 is not yet known, but one could speculate that, consistent with the Goldilocks principle, some complement activation is beneficial in the initial response to the virus, but too much, sustained for too long, propagates disease.

Through parallels with other coronaviruses, through systems biology approaches and through detailed pathological and clinical observations, researchers and clinicians now realize the importance of complement in pathophysiology and see the potential therapeutic benefit of complement inhibition ${ }^{6-9}$. Here we review the emerging roles of complement in COVID-19-associated thromboinflammation, propose a key contribution from lung intracellular complement as well as systemic complement activation, and finally provide an update on eagerly awaited clinical trial results.

\section{Complement in severe COVID-19}

Multiple lines of evidence implicate hyperactivation of the complement system in the pathophysiology of COVID-19. Mechanistically, SARS-CoV-2 itself can activate the complement system either directly through the lectin pathway, the classical pathway and/or the alternative pathway or cause endotheliopathy (endothelial cell injury and dysfunction) and thromboinflammation (inflammation associated with coagulation and thrombosis), which in turn activate the complement system (see later and FIG. 1). Specifically, the spike and nucleocapsid proteins of SARS-CoV-2 can be directly recognized by lectin pathway components, leading to complement activation ${ }^{10}$, and IgG and IgM antibodies directed against the receptor-binding domain of the spike protein initiate classical pathway activation ${ }^{11}$. Furthermore, SARS-CoV-2 spike protein may directly dysregulate the alternative pathway of complement activation by binding heparan sulfate and competing with factor $\mathrm{H}$, which is a negative regulator of complement activity ${ }^{12-14}$.

These observations are consistent with the long-known observation that complement activation is a frequent feature of the pathophysiology of acute respiratory distress syndrome, induced by infection or other triggers ${ }^{15-18}$. For example, increased anaphylatoxin concentrations are found in serum from patients with influenza virus H1N1 infection of the lungs ${ }^{19}$. Similarly, patients with severe COVID-19 have high circulating levels of C5a and of the terminal activation fragments, soluble C5b-9, which correlate with clinical severity ${ }^{11,20-22}$, as well as high levels of processed fragments of C3, itself an independent risk factor for death ${ }^{23}$. Likewise, data from the UK Biobank support genetic single-nucleotide variants in genes encoding the complement regulators decay accelerating factor (DAF; also known as CD55), complement factor $\mathrm{H}$ and complement component 4-binding protein- $\alpha(\mathrm{C} 4 \mathrm{BP} \alpha)$ as risk factors for morbidity and death from SARS-CoV-2 
infection $^{24}$. Significant microvascular deposition of mannan-binding lectin serine protease 2 (MASP2; the primary enzymatic initiator of the lectin pathway of complement activation), C4d and C5b-9 has been reported in the skin and lungs of patients, the last two complement components co-localizing with SARS-CoV-2 spike protein ${ }^{25,26}$. Reports of children with vasculitic lesions and hypercoagulability in the context of COVID-19 (REFS ${ }^{27,28}$ ) (the so-called multisystem inflammatory syndrome in children (MIS-C)) also suggest complement involvement through co-triggering of the complement and coagulation cascades. A key study has shown that elevated plasma levels of C5b-9 are common in MIS-C ${ }^{29}$, suggesting systemic complement activation in COVID-19. Characteristic endothelial cytomorphological changes observed in patients $^{30-32}$ are also pathognomonic of complement-mediated injury induced by C5b-9 $\left(\right.$ REF. $\left.^{33}\right)$. Finally, early clinical findings

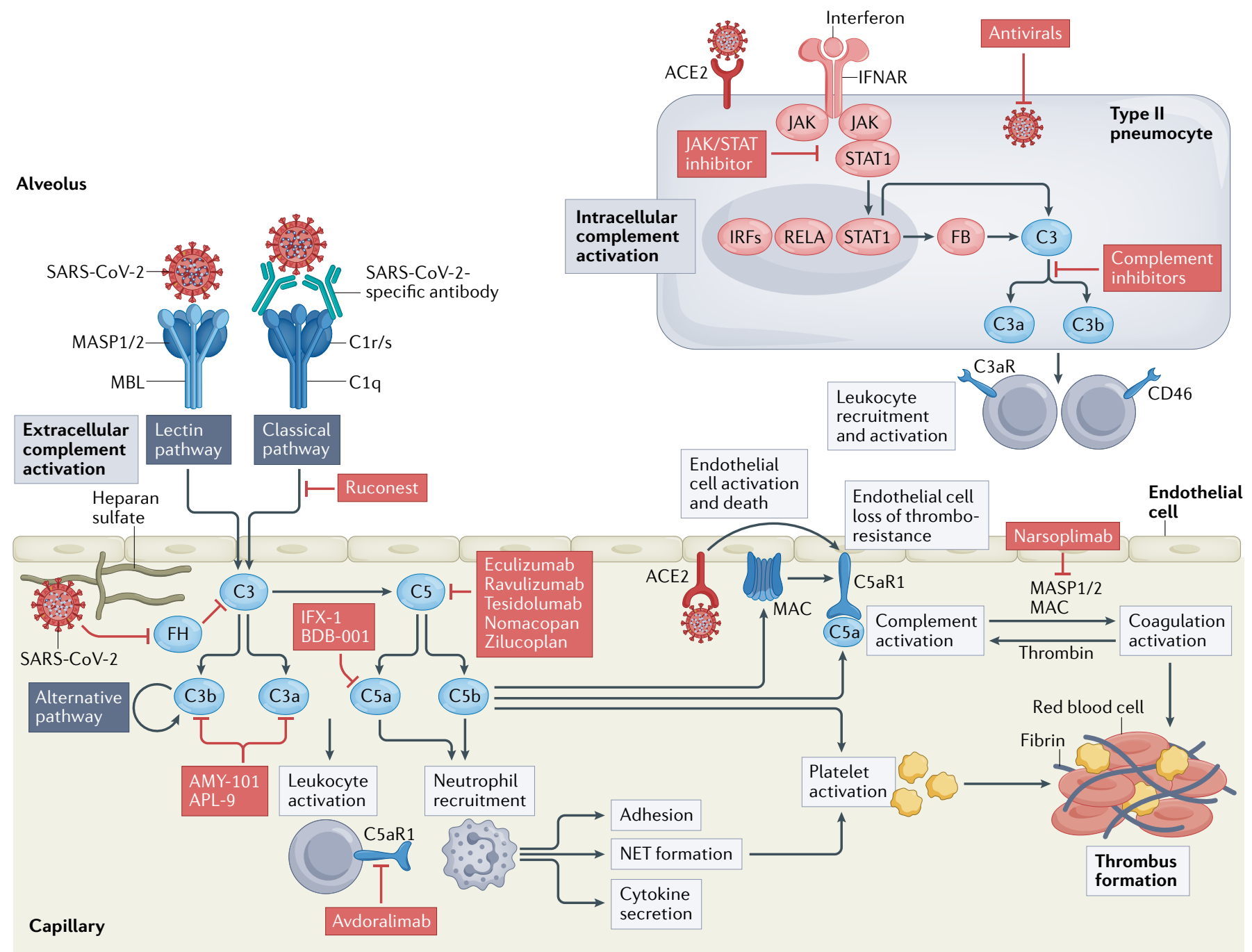

Fig. 1 | Complement as driver of and therapeutic target in COVID-19. Schematic summary of the currently known contributions of complement to COVID-19. Severe acute respiratory syndrome coronavirus 2 (SARS-CoV-2) triggers direct or indirect activation of extracellular and/or intracellular complement pathways. SARS-CoV-2 envelope proteins activate the lectin pathway through binding to mannose-binding lectin (MBL) or the classical pathway through SARS-CoV-2-specific antibodies and C1q. Competition of SARS-CoV-2 with C3b-regulatory factor $\mathrm{H}(\mathrm{FH})$ for binding to heparan sulfate removes the inhibitory effects of factor $\mathrm{H}$ on $\mathrm{C} 3$, sustaining activation of the alternative pathway. Infection-induced type I interferon receptor (IFNAR) signalling through the Janus kinase (JAK)-signalling transducer and activator of transcription 1 (STAT1) pathway in type Il pneumocytes induces production of intracellular complement factor $\mathrm{B}(\mathrm{FB})$ and $\mathrm{C} 3$, which seed an intracellular $\mathrm{C} 3$ convertase and cleavage-mediated activation of intracellular C3. Type II pneumocyte-generated C3 activation fragments engage cognate receptors ( $\mathrm{C} 3 \mathrm{a}-\mathrm{C} 3 \mathrm{aR}$ and $\mathrm{C} 3 \mathrm{~b}-\mathrm{CD} 46)$ on immune cells in the vicinity and induce their (hyper)activation. SARS-CoV-2-induced complement activation in the capillaries increases $\mathrm{C} 5$ a generation. $\mathrm{C} 5$ a potentiates activation of leukocytes and neutrophils, their adhesion to endothelial cells, their production of proinflammatory cytokines and/or the formation of local neutrophil extracellular traps (NETs). SARS-CoV-2-primed endothelial cells upregulate $\mathrm{C} 5$ a receptor 1 ( $\mathrm{C} 5 \mathrm{aR} 1$ ) expression and become vulnerable to pathological $\mathrm{C} 5 \mathrm{a}$ activation and insertion of the membrane attack complex (MAC), which cumulatively cause endothelial cell death and loss of thromboresistance. Simultaneously, complement induces platelet activation (mostly via action of the MAC) and activation of the coagulation cascade (mostly via action of MBL-associated serine protease 1 (MASP1) or MASP2), supporting detrimental thrombus formation. Current pharmacological interventions with specific complement targets are depicted. These include antivirals and JAK or STAT inhibitors, which reduce infection-induced extracellular and intracellular complement activation, and drugs that block MASP1 or MASP2 activity and classical pathway and C5 activation. Several therapeutics aim at inhibiting $\mathrm{C} 3$ and $\mathrm{C} 5$ activation fragments, and one inhibitory antibody blocks C5aR1 stimulation. ACE2, angiotensinconverting enzyme 2; IRF, interferon regulatory factor; RELA, nuclear factor-kB p65 subunit. 
and emerging trial evidence also indicate potential benefits of complement-targeting therapies in COVID-19, as reviewed later.

The findings of studies of patients align with findings in animals responding to related pandemic betacoronaviruses. For example, Middle East respiratory syndrome coronavirus infection in mice causes extensive complement deposition in lung tissues, where the damage can be reduced by inhibition of distal complement components $^{34}$. In the same model, C5aR blockade limits the thickening of alveolar septa and prevents interstitial oedema and haemorrhage in the lungs ${ }^{34}$. Similarly, the complement components $\mathrm{C} 3, \mathrm{Clr}$ and $\mathrm{Cfb}$ are all part of a pathogenic pulmonary gene signature associated with lethality in mouse models of SARS-CoV infection ${ }^{35}$, and deletion of $C 3$ in these mice protects against disease ${ }^{36}$. Indeed, primary infection with SARS-CoV upregulates complement genes in ferrets, including the lectin pathway components MASP1 and ficolin 1 (REF. ${ }^{37}$ ). SARS-CoV itself interacts with mannose-binding lectin (MBL) to activate complement $\mathrm{C} 3$ proximally via the lectin pathway on virus-infected cells ${ }^{38}$ in a similar manner to SARS-CoV-2, the nucleocapsid protein of which aggravates lung injury through MASP2-mediated complement hyperactivation ${ }^{10}$. The lectin pathway contributes to the excessive inflammatory response associated with other pandemic viral infections, as MBL-deficient mice develop less severe disease when infected with influenza A virus strain $\mathrm{H} 1 \mathrm{~N} 1$ or strain H9N2/G1 compared with wild-type mice ${ }^{39}$.

Although the detrimental contributions of complement to COVID-19 are now recognized, the underlying molecular mechanisms driving pathology are less clear. Recent data indicate that the long-acknowledged but still poorly understood functional connection between systemic complement and the coagulation cascade and also the intrinsic production of complement by lung cells are involved and may thus be promising novel therapeutic targets.

\section{Thromboinflammation in COVID-19}

SARS-CoV-2 infection of the respiratory epithelium triggers an inflammatory response driven by the host innate immune system that in $20-30 \%$ of hospitalized patients results in endothelial injury and the activation of coagulation and thrombosis ${ }^{40}$, a phenomenon called 'thromboinflammation' ${ }^{41}$. These events start in the lung, but may extend to several other organs, and can clinically manifest themselves as renal, hepatic and heart dysfunction and eventually multi-organ failure ${ }^{42,43}$. Thrombosis (venous thrombosis, pulmonary embolism and arterial thrombosis) is indeed a major complication of COVID-19. Despite routine thromboprophylaxis, almost $10 \%$ of patients hospitalized with COVID-19 have thrombotic complications ${ }^{44}$, and clotting-associated complications are associated with death ${ }^{45-50}$. Indeed, pulmonary artery thrombosis and microangiopathy in pulmonary tissue were observed in up to $79 \%$ of patients who died with COVID-19 as the primary cause and were associated with the presence of diffuse alveolar damage ${ }^{51}$, which supports the role of thrombotic phenomena in the development of COVID-19-related lung damage. Patients with COVID-19-associated coagulopathy have highly elevated levels of plasma D-dimer ${ }^{52}$, a fibrin degradation product that is present in the blood after activation of fibrinolysis. In a retrospective study of patients from Wuhan, China, D-dimer levels greater than $1 \mu \mathrm{g} \mathrm{ml}^{-1}$ at admission to hospital predicted an 18-fold increase in the odds of dying following SARS-CoV-2 infection ${ }^{2}$.

Endothelial cell injury and dysfunction (also termed 'endotheliopathy') play a central role in COVID-19-associated thrombosis and coagulopathy. Endothelial cells and microvascular pericytes express entry receptors for SARS-CoV-2 (REFS ${ }^{53-55}$ ), and endothelial tropism of SARS-CoV-2 has been suggested by autopsy studies and single-cell RNA sequencing atlases ${ }^{53,56}$. Here, SARS-CoV-2 binding to its host entry receptor, angiotensin-converting enzyme 2 (ACE2), blocks ACE2-mediated production of angiotensin (1-7), thus preventing vasorelaxation and antithrombotic and anti-inflammatory effects on endothelial cells and enhancing the harmful effects of angiotensin II, exacerbating endothelial cell activation and injury ${ }^{57}$. However, the evidence for direct SARS-CoV-2 infection of endothelial cells remains a matter of debate ${ }^{58-62}$. Nevertheless, the levels of markers of endothelial cell activation and injury, including von Willebrand factor (vWF), angiopoietin 2, soluble P-selectin and soluble thrombomodulin, are elevated in the circulation of patients with severe COVID-19 in intensive care units $^{63-65}$; and vWF and thrombomodulin levels correlate with mortality ${ }^{63,66,67}$.

Extensive endothelialitis with inflammatory cells adhering to the endothelium and platelet-rich fibrin thrombi have been reported in large and small arterial vessels in the lungs, heart, liver, small bowel, kidneys and other organs of patients who have succumbed to COVID-19 (REFS ${ }^{6,30,43}$ ). Furthermore, diffuse microvascular thrombosis with endothelial cell injury and rarefaction have been observed most often in pulmonary alveolar capillaries ${ }^{53}$, as well as in kidney glomerular and peritubular capillaries ${ }^{6,68,69}$ and in myocardial capillaries $^{43}$, accompanied by deposits of complement activation products within damaged microvasculature ${ }^{6,43,69}$.

Histological examination of lungs, hearts and kidneys from patients with severe COVID-19 shows fibrin and intense C5b-9 immunostaining in the glomeruli and cardiac microthrombi, respectively ${ }^{6,43,69}$. The pattern of microvascular lesions and C5b-9 deposits, along with elevated levels of lactate dehydrogenase and low levels of haptoglobin and schistocytes in peripheral blood, that have all been observed both in adults and children with severe COVID-19 (REFS ${ }^{51,53,70}$ ), are similar to complement-mediated thrombotic microangiopathy (also known as atypical haemolytic uraemic syndrome), a prototypic disease of complement C5-mediated endothelial injury $^{71,72}$. The report of two cases of atypical haemolytic uraemic syndrome relapse triggered by COVID-19, one of which was in a patient with a heterozygous missense variant in the $C 3$ gene (an R139W gain-of-function point mutation resulting in formation of a hyperactive C3 convertase), and cured by the anti-C5 antibody eculizumab ${ }^{73,74}$, indirectly supports a pathogenetic role of the complement terminal pathway in COVID-19-associated thromboinflammation.

The terminal complement components C5a and the MAC can promote endothelial injury and dysfunction through multiple processes $^{75}$. C5a recruits and activates neutrophils, monocytes and macrophages ${ }^{76}$. Furthermore, both C5a and the MAC induce chemokine release and upregulation of adhesion molecules on endothelial cells, events that support transmigration of neutrophils and macrophages ${ }^{75,77,78}$. C5a and the MAC also promote platelet adhesion by stimulating the exocytosis of P-selectin and vWF multimers from endothelial cells, tissue factor expression and shedding of thrombomodulin from cell surfaces ${ }^{76,79,80}$, which trigger the coagulation cascade. Activated platelets and C3 subsequently activate the recruited neutrophils to release neutrophil extracellular traps (NETs), generating extracellular DNA and histones that are highly toxic to endothelial cells and accumulate in microthrombi of patients with 
COVID-19 $\left(\right.$ REFS $\left.^{8,81}\right)$. The release of NETs (NETosis) is a unique form of neutrophil cell death that is intimately linked with the complement and coagulation cascades ${ }^{82}$. C5a receptor $1(\mathrm{C} 5 \mathrm{aR} 1)$ and $\mathrm{C} 5 \mathrm{aR} 2$ activation generates extracellular histones, which are major components of NETs and activate the intrinsic coagulation pathway ${ }^{83-85}$. NETs act as a scaffold for thrombus formation by inducing platelet aggregation, while platelets in turn induce NET formation ${ }^{86-88}$. The cumulative results of these events are vascular injury and loss of endothelial antithrombogenic properties, with massive formation of blood clots. Consistent with this, exposure of cultured microvascular endothelial cells to SARS-CoV-2 spike protein induced leukocyte recruitment, C3 and C5b-9 deposition and platelet adhesion and thrombus formation on cell surfaces. Thrombus formation was halted by complement inhibitors ${ }^{89}$. The observation that the signature of complement deposition across different organs in patients with severe COVID-19 mimics the signatures found in complement-driven thrombotic disease ${ }^{90}$ supports the notion that complement is a driving pathogenic force in severe COVID-19. Furthermore, it is possible that complement also contributes to SARS-CoV-2 vaccine-induced thrombotic complications (BOX 1).
Thus, collectively, the available evidence places the consequences of $\mathrm{C} 5$ activation among the drivers of COVID-19-associated thromboinflammation and highlights the potential of inhibitors targeting C5, C5a or C5aR1 to prevent and control thromboembolic complications in this debilitating infectious disease.

\section{Intracellular complement}

Complement circulating in plasma is produced and secreted by the liver ${ }^{91}$. However, complement components are also produced by both immune ${ }^{92,93}$ and non-immune ${ }^{94-96}$ cells. In T cells, complement is produced both on activation through the $\mathrm{T}$ cell receptor and via ICAM1-LFA1 interactions during diapedesis into tissues ${ }^{92,93}$. The latter scenario is relevant to COVID-19 as it represents a source of local complement in tissues, where plasma complement is absent, and is a requirement for the bioenergetic reprogramming necessary for optimal effector function of immune cells ${ }^{92,97-99}$. Airway epithelial cells (AECs) also produce $\mathrm{C} 3$ and have the capacity to take up some $\mathrm{C} 3$ from exogenous sources ${ }^{96}$. In the context of cellular stress, for example from serum starvation, intracellular C3 protects AECs from apoptosis ${ }^{96}$, although the mechanism is currently unknown.

\section{Box 1 | SARS-CoV-2 vaccine-induced thrombotic complications}

The development of vaccines against severe acute respiratory syndrome coronavirus 2 (SARS-CoV-2) has occurred with unprecedented speed, with at least three vaccines currently approved in most countries ${ }^{123,124}$ and more than six billion doses administered worldwide so far ${ }^{125}$. Overall, the risk of serious adverse effects has been remarkably low. However, several cases of unexpected thrombotic events and thrombocytopenia have been observed in individuals vaccinated with the adenovector-based vaccines ChAdOx1 nCoV-19 (AstraZeneca, University of Oxford and Serum Institute of India) ${ }^{126,127}$ and Ad26.COV2.S (Janssen; Johnson \& Johnson) ${ }^{128-130}$. Most affected patients developed acute disseminated intravascular coagulation, which can evolve into life-threatening cerebral venous thrombosis and thrombocytopenia following the first vaccination dose. The syndrome has been termed 'vaccine-induced immune thrombotic thrombocytopenia' (VITT) and is caused by high titres of circulating $\lg G$ autoantibodies that recognize platelet-bound platelet factor 4 (PF4; also known as CXCL4) and induce uncontrolled, Fc $\gamma$ lla-dependent platelet activation and uninhibited (local) coagulation ${ }^{126}$. Treatment of VITT includes use of non-heparin anticoagulants in combination with high-dose intravenous immunoglobulins (to competitively inhibit the Fc $\gamma$ lla receptor) ${ }^{131}$. VITT-related venous thrombosis is not confined to classical sites such as the lungs and deep veins of the leg but is often disseminated to the splanchnic, cerebral and ophthalmic veins ${ }^{132}$. The reasons for thrombosis at these uncommon locations in VITT are currently unclear. Although a role for complement in VITT has not been explored, there are several intriguing features of VITT that are suggestive of potential complement involvement. For example, as the main driver of VITT is a platelet-bound anti-PF4 IgG antibody, recognition of the $\lg \mathrm{G}$-platelet immune complexes by $\mathrm{C} 1 \mathrm{q}$ can initiate activation of the classical pathway. This could trigger subsequent activation of the alternative pathway and the coagulation system (FIG. 1) and create the thrombotic environment seen in VITT. Furthermore, the unusual locations of thrombotic precipitations observed in VITT are similar to those seen in paroxysmal nocturnal haemoglobinuria, a condition caused by overactive complement due to deficiency in the complement regulator CD59 (REFS ${ }^{133,134}$ ). Congruent with this, two patients with VITT improved after treatment with the anti-C5 antibody eculizumab ${ }^{135}$. However, although plausible, the potential contributions of complement to VITT are currently hypothetical and require further clinical and experimental exploration.

In the context of SARS-CoV-2 infection of AECs, expression of complement genes is among the biological pathways most highly induced by the virus ${ }^{100}$. Here, virus sensing triggers type I interferon signalling through the JAK-STAT machinery and induces direct regulation of complement genes, notably including $C 3$ and $C F B$ (encoding complement factor B) ${ }^{100}$. Factor B drives the assembly of an inducible, intracellular alternative complement activation convertase, which processes $\mathrm{C} 3$ to $\mathrm{C} 3 \mathrm{a}$. Accordingly, SARS-CoV-2-infected cells produce $\mathrm{C} 3 \mathrm{a}$, and this can be normalized by culture with ruxolitinib, an inhibitor of JAK1 and JAK2, or a cell-permeable inhibitor of factor $\mathrm{B}^{100}$. Importantly, immune cells close to SARS-CoV-2-infected AECs respond to the milieu of heightened local C3 activation and upregulate genes induced by C3 fragment receptors (C3aR and CD46), generating proinflammatory mediators, a signature not detected in circulating immune cells ${ }^{100}$. Whether increased C3 production and intracellular $\mathrm{C} 3 \mathrm{a}$ generation by AECs triggered by SARS-CoV-2 infection has a cell-intrinsic effect has not yet been explored. Collectively, AEC-derived complement is likely to represent a substantial local source of complement in the context of SARS-CoV-2 and is unlikely to be normalized by drugs targeting complement components in the circulation.

Given the contributions of canonical (plasma-circulating) and non-canonical (intracellular) complement to human immunological health ${ }^{101,102}$, it may be worth exploring whether changes in complement activity with age may be a risk factor for severe COVID-19 (BOX 2).

\section{Therapeutic perspectives}

Early in the first wave of the COVID-19 pandemic, a number of case reports and small-scale clinical interventions that were not randomized nor properly controlled advocated the use of complement-inhibiting therapies ${ }^{103-109}$, very similar to reports advocating the wide-scale use of cytokine antagonists ${ }^{110}$. What has become clear over the past 18 months is that initial enthusiasm needs to be followed by rigorously executed clinical trials of appropriate sample size across many countries before conclusions can be reached and clinical practice is altered. For COVID-19 therapy, it is emerging that timing is everything. The disease runs a bimodal course with an early viral phase comprising mainly upper airway symptoms, followed in approximately $20 \%$ of patients by dyspnea and hypoxia, sometimes progressing to severe acute respiratory 
distress syndrome. Despite the considerable numbers of patients who are eligible and willing to participate in clinical COVID-19 trials, only $20 \%$ of patients progress to severe disease. Thus, the biggest hurdle to obtaining definitive answers with various intervention trials in severe COVID-19 has been to select the right timing and the right patient who might benefit from the intervention, on the basis of either clinical or biochemically easy to measure (point of care) biomarkers. It is very likely that the hyperinflammation that characterizes severe disease is driven by several molecular and cellular pathways that tend to self-amplify ${ }^{111}$, so in advanced disease, targeting only one pathway and hoping for improvement is likely to be futile. Early intervention without causing immunosuppression is probably key. Currently, however, biomarkers predicting those who will go on to develop life-threatening COVID-19 (prognostic biomarkers) among the less ill and that could predict those who will respond to therapy (theragnostic biomarkers) are still lacking. One notable exception is the serum concentration of soluble urokinase plasminogen activator receptor, which strongly predicts clinical responsiveness to blockade with the IL-1 receptor antagonist anakinra ${ }^{112}$. In the specific case of complement inhibition studies, it is still complicated in most hospitals to get point-of-care assessments of the complement cascade and in a timely manner. Clinical practice is also rapidly changing during this pandemic, and use of the broad anti-inflammatory drug dexamethasone has become the standard of care, potentially making it harder to replicate earlier findings regarding new compounds in small-scale studies in larger randomized trials that now include dexamethasone as the standard of care. As an example, despite many negative clinical outcome trials, the effectiveness of interfering with IL-6 in COVID-19 became clear only from a meta-analysis of more than 30 randomized intervention trials, in which it was shown that IL-6 reduces 28 -day mortality mainly in the most severely ill patients ${ }^{113}$. Counterintuitively, the clinical benefit was, however, seen mainly in those receiving dexamethasone or other steroids, potentially because these patients had a worse prognosis ${ }^{114,115}$. Specifically, with regard to complement, other considerations include whether components of the complement system themselves should be targeted (extracellularly or within cells) or whether mechanisms inducing complement production and/or activation should be targeted instead (for example, using

\section{Box 2 | Risk factors for severe COVID-19}

One of the major unanswered questions of the COVID-19 pandemic is why some people succumb to the disease while others experience only mild symptoms following infection. Surveillance data indicate that older adults (over 70 years of age) and those with underlying health conditions have an increased risk of progression to severe COVID-19 (REF. $\left.{ }^{136}\right)$. Specifically, co-morbidities such as diabetes, heart disease and hypertension (which are progressively more prevalent with age), obesity and smoking are associated with severe disease or fatal outcomes ${ }^{137}$. The underlying molecular mechanisms that render these subgroups of patients more vulnerable are a focus of ongoing research - and complement perturbations should be considered as potential candidates. Complement plays an active role in the development of hypertension ${ }^{138}$ and cardiovascular disease ${ }^{139}$, while smoking and obesity are both associated with chronically increased complement activation, which perpetuates the associated diseases ${ }^{140,141}$. Thus, complement contributes to the pathological conditions priming individuals to development of severe COVID-19. More importantly, complement may also contribute to the changes associated with the ageing immune system, referred to as 'immunosenescence" ${ }^{\text {"142 }}$, and that augment vulnerability towards severe COVID-19 in otherwise healthy individuals ${ }^{143}$. Specifically, the continued presence of low-grade inflammation (inflammageing) over a lifetime can fuel the onset or progression of age-related pathological inflammation and autoimmunity. Indeed, the protein levels of classical and alternative pathway complement components in the circulation increase progressively with age ${ }^{144}$, and increases in the amounts of circulating $\mathrm{C} 3$ and $\mathrm{C} 1 \mathrm{q}$ proteins are considered biomarker candidates of ageing and age-related diseases ${ }^{145}$. Furthermore, increases in complement production in the brain contribute to age-related synaptic loss and microglia dysfunction ${ }^{146}$, whereas an age-related increase in C1q protein levels in circulation impairs muscle regeneration over time ${ }^{147}$. Thus, it is feasible that age-related changes in the amounts of specific complement proteins contribute to the detrimental imbalance in proinflammatory versus repair responses disproportionately observed in older patients (over 70 years of age) with COVID-19.

JAK inhibitors or factor B inhibitors; see earlier). These considerations are important because AEC-derived complement is likely to represent a substantial local source of complement in the context of SARS-CoV-2 and is unlikely to be normalized by drugs targeting complement components in the circulation. Cell-permeating drugs targeting complement intracellularly (such as factor B inhibitors) or upstream components inducing complement gene transcription (such as JAK inhibitors) could potentially be more efficacious in severe COVID-19. Indeed, although many trials are ongoing, some data already support beneficial efficacy of JAK inhibitors in the context of severe COVID-19 ${ }^{116-119}$. Finally, although the proliferation of preprint publications somewhat mitigates publication bias, it still remains likely that negative data may be under-reported.

Strategies to target complement in COVID-19 have mainly used available drugs that were already undergoing clinical testing or were already approved for clinical use in complement-mediated diseases, such as thrombotic microangiopathy associated with transplantation, atypical haemolytic uraemic syndrome, paroxysmal nocturnal haemoglobinuria, macular degeneration, complement-mediated nephropathies and myasthenia gravis. As a result, most of these have focused on targeting the C5 system. Initially, a few case series of C5-targeting therapies (eculizumab, a monoclonal antibody targeting $\mathrm{C} 5$, by Alexion
Pharmaceuticals; BDB-001, a monoclonal antibody against $\mathrm{C} 5 \mathrm{a}$, by Staidson Biopharmaceuticals) were trialled in a total of 16 patients with severe to critical COVID-19, 14 of whom recovered ${ }^{26,106,109}$. A subsequent proof-of-concept nonrandomized trial in 40 patients with severe COVID-19 and controls showed a survival benefit in the group treated in an intensive care unit setting with eculizumab $^{120}$. On the basis of these studies, several randomized controlled trials (RCTs) have been initiated, and some have completed enrolment. Targeting C5 in COVID-19 has been trialled using the monoclonal antibodies eculizumab (NCT04288713 and NCT04346797), ravulizumab (Ultomiris; NCT04570397 and NCT04369469) and tesidolumab (LFG316, Novartis) ${ }^{108}$, or using small protein or peptide antagonists of C5 such as nomacopan (Akari Therapeutics; trial about to start in the UK and Brazil) or zilucoplan (ZILU-COV trial (NCT04382755, UCB Pharma). Interim analysis of a large phase III trial with ravulizumab (NCT04369469) in patients with COVID-19 already receiving mechanical ventilation at randomization could not demonstrate clinical benefit, leading to a pause in the study. In the ZILU-COV trial, in contrast, 81 patients with COVID-19 and signs of hypoxia not yet requiring mechanical ventilation were randomized to receive $32 \mathrm{mg}$ of zilucoplan subcutaneous daily for 14 days in addition to prophylactic broad-spectrum antibiotics 
(to cover the risk of meningococcal disease) or standard-of-care treatment and the same antibiotic regimen ${ }^{121}$. Patients receiving zilucoplan had improved oxygenation parameters at day 15, accompanied by a lowering of circulating cytokine levels. At day 28 there was also lower mortality in zilucoplan-treated patients, although the study was underpowered to detect effects on mortality. Importantly, there was no risk of opportunistic infections in those receiving zilucoplan. This indicates that early timing of C5 blockade may be crucial. Trials are also studying the impact of blocking either C5a or C5aR. An initial RCT using the C5a-targeting antibody vilobelimab (NCT04333420, InflaRx) reported minimal improvement in oxygenation in 30 patients and controls, although there was a trend for increased survival in the vilobelimab $\operatorname{arm}^{122}$. This study has now been extended to a large multicentre placebo-controlled RCT involving 390 patients receiving mechanical ventilation (NCT04333420) and is currently recruiting patients. Similarly, a large phase II/III RCT involving 368 participants is addressing the C5a-specific antibody BDB-001 (NCT 04449588, Staidson Biopharmaceuticals) and is in progress. Despite promising results of C5aR blockade in a preclinical model ${ }^{20}$, development of the C5aR1-specific monoclonal antibody avdoralimab (Innate Pharma, NCT04371367) was discontinued after disappointing effects of a phase II RCT in patients with various degrees of COVID-19 severity. One explanation is that blockade of C5aR1 alone is not sufficient to halt the detrimental effects of complement in COVID-19 because the MAC is still formed or because $\mathrm{C} 5$ can still signal through alternative receptors, such as $\mathrm{C} 5 \mathrm{aR} 2$.

Targeting the $\mathrm{C} 3$ pathway is also an attractive scenario. Use of the C3 inhibitor AMY-101 (Amyndas Pharma) was initially reported in two case reports for treatment of four patients with severe COVID-19, all of whom subsequently recovered ${ }^{105,106}$. A larger phase II study with 144 patients (NCT04395456) is planned but is not yet recruiting. The C3 antagonist APL9 (Apellis, NCT04402060) was discontinued after no difference in mortality was found in a phase I/II RCT in 65 patients with severe COVID-19. Other strategies of which the outcome is not yet known include blocking complement early in the cascade, such as by using the recombinant human $\mathrm{C} 1$ inhibitor Ruconest (Pharming, NCT04705831, NCT0530136 and NCT04414631) or the MASP2-specific antibody narsoplimab (Omeros, NCT04488081) to block the lectin pathway of complement activation. Similarly, given the potential for SARS-CoV-2 spike protein to activate complement via the alternative pathway ${ }^{12-14}$, a phase II clinical trial of remdesivir, with or without a factor $\mathrm{D}$ inhibitor, is currently under way (Alexion, NCT04988035).

An important cautionary note that also needs to be considered in the context of strategies that inhibit a non-redundant component of normal immunity is the risk of life-threatening, invasive infections that are distinct from COVID-19 itself. As examples, a fatal case of Klebsiella pneumoniae infection following compassionate use of a C5-blocking monoclonal antibody in one of five patients ${ }^{108}$ and a more than doubling of infectious complications, including bacteraemia and ventilator-associated pneumonia, in a clinical trial of eculizumab have been reported ${ }^{120}$. These infectious complications occurred despite adequate prior antibiotic prophylaxis and vaccination. Thus, the potential gains of complement inhibition need also to be balanced against the potential risks and harms that may also ensue.

\section{Conclusions}

Both clinical and basic science studies suggest that uncontrolled activity of the complement system may be a central player in the pathogenesis of COVID-19. Clinical trial evidence is now beginning to substantiate this suspicion. This clearly is an exciting development; however, it should still be viewed with caution because, similarly to IL-6-targeting therapies, it is likely that clinical trials will indicate that the benefits of complement-targeting therapies will depend on several confounders, namely severity of disease, timing of initiation and co-administered drugs. Thus, at present there is no compelling evidence to strongly advocate complement-targeting treatments for all patients presenting with severe COVID-19. This will likely change in the future as the niche for complement targeting becomes clearer and as we gain a better understanding of the distinct spatio-temporal contributions of complement following SARS-CoV-2 infection, its crosstalk with the coagulation system and the role of genetic polymorphisms in both systems.

\section{Behdad Afzali iD ${ }^{1 凶}$, Marina Noris (iD ${ }^{2,3}$,} Bart N. Lambrecht ${ }^{4,5,6 \times}$ and Claudia Kemper (iD) ${ }^{7,8} \times$

'Immunoregulation Section, Kidney Diseases Branch, National Institute of Diabetes and Digestive and Kidney Diseases, National Institutes of Health, Bethesda, MD, USA.
2Istituto di Ricerche Farmacologiche "Mario Negri", Clinical Research Center for Rare Diseases "Aldo e Cele Daccō", Ranica, Italy.

3"Centro Anna Maria Astori”, Bergamo, Italy.

${ }^{4}$ Laboratory of Immunoregulation and Mucosal Immunology, VIB-UGent Center for Inflammation Research, Ghent, Belgium.

${ }^{5}$ Department of Internal Medicine and Pediatrics, Ghent University, Ghent, Belgium.

${ }^{6}$ Department of Pulmonary Medicine, Erasmus University Medical Center, Rotterdam, Netherlands.

${ }^{7}$ Complement and Inflammation Research Section (CIRS), National Heart, Lung, and Blood Institute, National Institutes of Health, Bethesda, MD, USA.

${ }^{8}$ Institute for Systemic Inflammation Research, University of Lübeck, Lübeck, Germany.

凶e-mail:ben.afzali@nih.gov; marina.noris@ marionegri.it; bart.lambrecht@ugent.be; claudia. kemper@nih.gov

https://doi.org/10.1038/s41577-021-00665-1

Published online 15 December 2021

1. Dong, E., Du, H. \& Gardner, L. An interactive web-based dashboard to track COVID-19 in real time. Lancet Infect. Dis. 20, 533-534 (2020).

2. Zhou, F. et al. Clinical course and risk factors for mortality of adult inpatients with COVID-19 in Wuhan, China: a retrospective cohort study. Lancet 395 . 1054-1062 (2020).

3. Whitaker, M. et al. Persistent symptoms following SARS-CoV-2 infection in a random community sample of 508,707 people. Preprint at medRxiv https:// doi.org/10.1101/2021.06.28.21259452 (2021).

4. Vasarmidi, E., Tsitoura, E., Spandidos, D. A Tzanakis, N. \& Antoniou, K. M. Pulmonary fibrosis in the aftermath of the COVID-19 era (review). Exp. Ther. Med. 20, 2557-2560 (2020).

5. Noris, M. \& Remuzzi, G. Overview of complement activation and regulation. Semin. Nephrol. 33, 479-492 (2013).

6. Noris, M., Benigni, A. \& Remuzzi, G. The case of complement activation in COVID-19 multiorgan impact. Kidney Int. 98, 314-322 (2020).

7. Lo, M. W., Kemper, C. \& Woodruff, T. M. COVID-19: complement, coagulation, and collateral damage. J. Immunol. 205, ji2000644 (2020).

8. Skendros, P. et al. Complement and tissue factorenriched neutrophil extracellular traps are key drivers in COVID-19 immunothrombosis. J. Clin. Invest. 130 6151-6157 (2020).

9. Perico, L. et al. Immunity, endothelial injury and complement-induced coagulopathy in COVID-19. Nat. Rev. Nephrol. 17, 46-64 (2021).

10. Ali, Y. M. et al. Lectin pathway mediates complement activation by SARS-CoV-2 proteins. Front. Immunol. 12, 714511 (2021)

This study provides the first demonstration that SARS-CoV-2 directly activates complement via MASP1 and MASP2

11. Holter, J. C. et al. Systemic complement activation is associated with respiratory failure in COVID-19 hospitalized patients. Proc. Natl Acad. Sci. USA 117, 25018-25025 (2020)

12. Yu, J. et al. Complement dysregulation is associated with severe COVID-19 illness. Haematologica https:// doi.org/10.3324/haematol.2021.279155 (2021).

13. $\mathrm{Yu}, \mathrm{J}$. et al. Direct activation of the alternative complement pathway by SARS-CoV- 2 spike proteins is blocked by factor $\mathrm{D}$ inhibition. Blood 136 2080-2089 (2020).

14. Satyam, A. et al. Activation of classical and alternative complement pathways in the pathogenesis of lung injury in COVID-19. Clin. Immunol. 226, 108716 (2021).

15. Robbins, R. A Russ, W. D Rasmussen, J. K. \& Clayton, M. M. Activation of the complement system in the adult respiratory distress syndrome $1-4$. Am. Rev. Respir. Dis. 135, 651-658 (1987).

16. Zilow, G., Sturm, J. A., Rother, U. \& Kirschfink, M Complement activation and the prognostic value of $\mathrm{C} 3 \mathrm{a}$ in patients at risk of adult respiratory distress syndrome. Clin. Exp. Immunol. 79, 151-157 (1990).

17. Zilow, G., Joka, T., Obertacke, U., Rother, U. \& Kirschfink, M. Generation of anaphylatoxin C3a in 
plasma and bronchoalveolar lavage fluid in trauma patients at risk for the adult respiratory distress syndrome. Crit. Care Med. 20, 468-473 (1992).

18. Duchateau, J. et al. Complement activation in patients at risk of developing the adult respiratory distress syndrome 1-3. Am. Rev. Respir. Dis. 130, 1058-1064 (1984).

19. Ohta, R. et al. Serum concentrations of complement anaphylatoxins and proinflammatory mediators in patients with $2009 \mathrm{H} 1 \mathrm{~N} 1$ influenza. Microbiol. Immunol. 55, 191-198 (2011).

20. Carvelli, J et al. Association of COVID-19 inflammation with activation of the C5a-C5aR 1 axis. Nature 588, 146-150 (2020) This is a key publication demonstrating that increased $\mathrm{C} 5 \mathrm{a}$ levels in the circulation and hyperexpression and hyperactivation of C5aR1 on blood and pulmonary immune cells contribute to COVID-19 severity

21. Cugno, M. et al. Complement activation in patients with COVID-19: a novel therapeutic target. J. Allergy Clin. Immun. 146, 215-217 (2020).

22. Shen, B. et al. Proteomic and metabolomic characterization of COVID-19 patient sera. Cell 182 59-72.e15 (2020)

This is a fundamental data source allowing novel insights into disease mechanisms of COVID-19 beyond the battery of single-cell RNA-sequencing data available.

23. Sinkovits, G. et al. Complement overactivation and consumption predicts in-hospital mortality in SARSCoV-2 infection. Front. Immunol. 12, 663187 (2021).

24. Ramlall, V. et al. Immune complement and coagulation dysfunction in adverse outcomes of SARS-CoV-2 infection. Nat. Med. 26, 1609-1615 (2020).

25. Magro, C. et al. Complement associated microvascular injury and thrombosis in the pathogenesis of severe COVID-19 infection: a report of five cases. Transl. Res. 220, 1-13 (2020).

26. Gao, T. et al. Highly pathogenic coronavirus $\mathrm{N}$ protein aggravates lung injury by MASP-2-mediated complement over-activation. Preprint at medRxiv https://doi.org/10.1101/2020.03.29.20041962 (2020).

27. Riphagen, S., Gomez, X., Gonzalez-Martinez, C. Wilkinson, N. \& Theocharis, P. Hyperinflammatory shock in children during COVID-19 pandemic. Lancet 395, 1607-1608 (2020)

28. Verdoni, L. et al. An outbreak of severe Kawasaki-like disease at the Italian epicentre of the SARS-CoV-2 epidemic: an observational cohort study. Lancet 395 . 1771-1778 (2020)

This is among the first studies showing that SARS-CoV-2 can induce a spectrum of multisystem inflammatory syndromes.

29. Syrimi, E. et al. The immune landscape of SARS-CoV-2 associated multisystem inflammatory syndrome in children (MIS-C) from acute disease to recovery. iscience 24, 103215 (2021)

30. Varga, Z. et al. Endothelial cell infection and endotheliitis in COVID-19. Lancet 395, 1417-1418 (2020).

31. Su, H. et al. Renal histopathological analysis of 26 postmortem findings of patients with COVID-19 in China. Kidney Int. 98, 219-227 (2020).

32. Bryce, $\mathrm{C}$. et al. Pathophysiology of SARS-CoV-2: targeting of endothelial cells renders a complex disease with thrombotic microangiopathy and aberrant immune response. The Mount Sinai COVID-19 autopsy experience. Preprint at medRxiv https://doi.org/10.1101/2020.05.18.20099960 (2020).

33. Timmermans, S. A. M. E. G. et al. C5b9 formation on endothelial cells reflects complement defects among patients with renal thrombotic microangiopathy and severe hypertension. J. Am. Soc. Nephrol. 29 2234-2243 (2018)

34. Jiang, $\mathrm{Y}$. et al. Blockade of the $\mathrm{C} 5 \mathrm{a}-\mathrm{C} 5 \mathrm{aR}$ axis alleviates lung damage in hDPP4-transgenic mice infected with MERS-CoV. Emerg. Microbes Infec 7, 77 (2018)

35. Rockx, B. et al. Early upregulation of acute respiratory distress syndrome-associated cytokines promotes lethal disease in an aged-mouse model of severe acute respiratory syndrome coronavirus infection. J. Virol. 83, 7062-7074 (2009).

36. Gralinski, L. E. et al. Complement activation contributes to severe acute respiratory syndrome coronavirus pathogenesis. mBio 9, e01753-18 (2018).

This study provides the first evidence in a smal animal in vivo model that complement activation contributes to SARS-CoV-2-induced airway pathology by demonstrating that C3-deficient, virus-infected mice have less pathology compared with wild-type mice.

37. Cameron, M. J. et al. Lack of innate interferon responses during SARS coronavirus infection in a vaccination and reinfection ferret model. PLOS ONE 7 e45842 (2012).

38. Ip, W. K. E. et al. Mannose-binding lectin in severe acute respiratory syndrome coronavirus infection. J. Infect. Dis. 191, 1697-1704 (2005).

39. Ling, M. T. et al. Mannose-binding lectin contributes to deleterious inflammatory response in pandemic $\mathrm{H} 1 \mathrm{~N} 1$ and avian H9N2 infection. J. Infect. Dis. 205, 44-53 (2012).

40. Middeldorp, S. et al. Incidence of venous thromboembolism in hospitalized patients with COVID-19. J. Vasc. Surg. Venous Lymphatic Disord. 9 536 (2021).

41. Iffah, R. \& Gavins, F. N. E. Thromboinflammation in coronavirus disease 2019: the clot thickens. Brit J. Pharmacol. https://doi.org/10.1111/bph. 15594 (2021).

42. Wadman, M., Couzin-Frankel, J., Kaiser, J. \& Matacic, C. A rampage through the body. Science 368, 356-360 (2020).

43. Pellegrini, D. et al. Microthrombi as a major cause of cardiac injury in COVID-19: a pathologic study. Circulation 143, 1031-1042 (2021).

44. Al-Samkari, H. et al. COVID-19 and coagulation: bleeding and thrombotic manifestations of SARS CoV-2 infection. Blood 136, 489-500 (2020).

45. Pujhari, S., Paul, S., Ahluwalia, J. \& Rasgon, J. L. Clotting disorder in severe acute respiratory syndrome coronavirus 2. Rev. Med. Virol. 31, e2177 (2021).

46. Lax, S. F. et al. Pulmonary arterial thrombosis in COVID-19 with fatal outcome: results from a prospective, single-center, clinicopathologic case series. Ann. Intern. Med. 173, 350-361 (2020).

47. Shi, J. et al. Coagulation dysfunction in ICU patients with coronavirus disease 2019 in Wuhan, China: a retrospective observational study of 75 fatal cases. Aging 13, 1591-1607 (2020).

48. Violi, F. et al. Arterial and venous thrombosis in coronavirus 2019 disease (Covid-19): relationship with mortality. Intern. Emerg. Med. 16, 1231-1237 (2021).

49. Pérez-García, F. et al. Age-adjusted endothelial activation and stress index for coronavirus disease 2019 at admission is a reliable predictor for 28-day mortality in hospitalized patients with coronavirus disease 2019. Front. Med. 8, 736028 (2021).

50. Contou, D. et al. Causes and timing of death in critically ill COVID-19 patients. Crit. Care 25, 79 (2021).

51. Romanova, E. S. et al. Cause of death based on systematic post-mortem studies in patients with positive SARS-CoV-2 tissue PCR during the COVID-19 pandemic. J. Intern. Med. 290, 655-665 (2021).

52. Spiezia, L et al COVID-19-related severe hypercoagulability in patients admitted to intensive care unit for acute respiratory failure. Thromb. Haemost. 120, 998-1000 (2020)

53. Ackermann, M. et al. Pulmonary vascular endothelialitis, thrombosis, and angiogenesis in Covid-19. N. Engl. J. Med. 383, 120-128 (2020).

54. Aimes, R. et al. Endothelial cell serine proteases expressed during vascular morphogenesis and angiogenesis. Thromb. Haemost. 89, 561-572 (2003).

55. Nicin, L. et al. Cell type-specific expression of the putative SARS-CoV-2 receptor ACE2 in human hearts. Eur. Heart J. 41, 1804-1806 (2020)

56. Delorey, T. M. et al. COVID-19 tissue atlases reveal SARS-CoV-2 pathology and cellular targets. Nature 595, 107-113 (2021).

57. Vinci, R. et al. From angiotensin-converting enzyme 2 disruption to thromboinflammatory microvascular disease: a paradigm drawn from COVID-19. Int. J. Cardiol. 326, 243-247 (2021)

58. Monteil, V. et al. Inhibition of SARS-CoV-2 infections in engineered human tissues using clinical-grade soluble human ACE2. Cell 181, 905-913.e7 (2020).

59. Wang, P. et al. A cross-talk between epithelium and endothelium mediates human alveolar-capillary injury during SARS-CoV-2 infection. Cell Death Dis. 11, 1042 (2020).

60. Bryce, C. et al. Pathophysiology of SARS-CoV-2: the Mount Sinai COVID-19 autopsy experience. Mod. Pathol. 34, 1456-1467 (2021)

61. Sia, S. F. et al. Pathogenesis and transmission of SARS-CoV-2 in golden hamsters. Nature 583, 834-838 (2020)

With this publication, the authors provide a hamster in vivo model of mild SARS-CoV-2 infection which mimics human disease better than comparable mouse models.

62. Schimmel, L. et al. Endothelial cells are not productively infected by SARS-CoV-2. Clin. Transl. Immunol. 10, e1350 (2021).

63. Goshua G et al. Endotheliopathy in COVID-19associated coagulopathy: evidence from a singlecentre, cross-sectional study. Lancet Haematol. 7 , e575-e582 (2020).

64. O'Sullivan, J. M., Gonagle, D. M., Ward, S. E., Preston, R. J. S. \& O’Donnell, J. S. Endothelial cells orchestrate COVID-19 coagulopathy. Lancet Haematol. 7, e553-e555 (2020).

65. Ma, L. et al. Increased complement activation is a distinctive feature of severe SARS-CoV-2 infection. Sci. Immunol. 6, eabh2259 (2021).

66. Cotter, A. H., Yang, S.-J. T., Shafi, H., Cotter, T. M. \& Palmer-Toy, D. E. Elevated von Willebrand factor antigen is an early predictor of mortality and prolonged length of stay for coronavirus disease 2019 (COVID-19) inpatients. Arch. Pathol. Lab. Med. https://doi.org/10.5858/arpa.2021-0255-sa (2021).

67. Marchetti, M. et al. Endothelium activation markers in severe hospitalized COVID-19 patients: role in mortality risk prediction. TH Open 5, e253-e263 (2021).

68. Fox, S. E. et al. Pulmonary and cardiac pathology in African American patients with COVID-19: an autopsy series from New Orleans. Lancet Respir. Med. 8 , 681-686 (2020)

69. Pfister, F. et al. Complement activation in kidneys of patients with COVID-19. Front. Immunol. 11, 594849 (2021).

70. Diorio $\mathrm{C}$ et al. Evidence of thrombotic microangiopathy in children with SARS-CoV-2 across the spectrum of clinical presentations. Blood Adv. $\mathbf{4}$, 6051-6063 (2020)

71. Noris, M., Mescia, F \& Remuzzi, G. STEC-HUS, atypical HUS and TTP are all diseases of complement activation. Nat. Rev. Nephrol. 8, 622-633 (2012).

72. Noris, M. et al. Dynamics of complement activation in aHUS and how to monitor eculizumab therapy. Blood 124, 1715-1726 (2014).

73. Mat, O. et al. Kidney thrombotic microangiopathy after COVID-19 associated with C3 gene mutation. Kidney Int. Rep. 6, 1732-1737 (2021).

74. Ville, S. et al. Atypical HUS relapse triggered by Covid-19. Kidney Int. 99, 267-268 (2020).

75. Riedl, M. et al. Complement activation induces neutrophil adhesion and neutrophil-platelet aggregat formation on vascular endothelial cells. Kidney Int. Rep. 2, 66-75 (2017).

76. Skeie, J. M., Fingert, J. H., Russell, S. R., Stone, E. M. \& Mullins, R. F. Complement component C5a activates ICAM-1 expression on human choroidal endothelial cells. Invest. Ophth Vis. Sci. 51, 5336-5342 (2010).

77. Kilgore, K. S., Ward, P. A. \& Warren, J. S. Neutrophil adhesion to human endothelial cells is induced by the membrane attack complex: the roles of P-selectin and platelet activating factor. Inflammation 22, 583-598 (1998).

This is pioneering work connecting MAC formation with endothelial cell and platelet activation

78. Foreman, K. E. et al. C5a-induced expression of P-selectin in endothelial cells. J. Clin. Invest. 94, 1147-1155 (1994).

79. Ikeda, K. et al. C5a induces tissue factor activity on endothelial cells. Thromb. Haemost. 77, 394-398 (1997).

80. Bettoni, S. et al. Interaction between multimeric von Willebrand factor and complement: a fresh look to the pathophysiology of microvascular thrombosis. J. Immunol. 199, 1021-1040 (2017).

81. Leppkes, M. et al. Vascular occlusion by neutrophil extracellular traps in COVID-19. Ebiomedicine 58, 102925 (2020)

82. Bont, C. M., de, Boelens, W. C. \& Pruijn, G. J. M. NETosis, complement, and coagulation: a triangular relationship. Cell Mol Immunol 16, 19-27 (2019).

83. $\mathrm{Xu}, \mathrm{J}$. et al. Extracellular histones are major mediators of death in sepsis. Nat. Med. 15, 1318-1321 (2009).

84. Semeraro, F. et al. Extracellular histones promote thrombin generation through platelet-dependent mechanisms: involvement of platelet TLR2 and TLR4. Blood 118, 1952-1961 (2011).

85. Bosmann, M. et al. Extracellular histones are essential effectors of C5aR- and C5L2-mediated tissue damage and inflammation in acute lung injury. FASEB J. 27. 5010-5021 (2013).

This is the first study to connect local histone $\mathrm{H} 3$ and $\mathrm{H} 4$ release as DAMPs that drive $\mathrm{C5}$ activation and subsequent induction of tissue pathologies in rat and mouse models of acute lung injury. 
86. Caudrillier, A. et al. Platelets induce neutrophil extracellular traps in transfusion-related acute lung injury. J. Clin. Invest. 122, 2661-2671 (2012).

87. Middleton, E. A. et al. Neutrophil extracellular traps contribute to immunothrombosis in COVID-19 acute respiratory distress syndrome. Blood 136 1169-1179 (2020). This study is among the first to demonstrate that neutrophils and specifically neutrophil NETs contribute to COVID-19 pathology.

88. Barnes, B. J. et al. Targeting potential drivers of COVID-19: neutrophil extracellular traps. J. Exp. Med. 217, e20200652 (2020).

89. Perico, L. et al. SARS-CoV-2 spike protein 1 activates microvascular endothelial cells and complement system leading to thrombus formation. SSRN Electron. J. https://doi.org/10.2139/ssrn.3864027 (2021).

90. Macor, P. et al. Multiple-organ complement deposition on vascular endothelium in COVID-19 patients. Biomed 9, 1003 (2021)

91. Walport, M. J. Complement. First two parts. N. Engl. J. Med. 344, 1058-1066 (2001)

92. Kolev, M. et al. Diapedesis-induced integrin signaling via LFA- 1 facilitates tissue immunity by inducing intrinsic complement $\mathrm{C} 3$ expression in immune cells. Immunity 52, 513-527.e8 (2020).

93. Liszewski, M. K. et al. Intracellular complement activation sustains $\mathrm{T}$ cell homeostasis and mediates effector differentiation. Immunity 39, 1143-1157 (2013)

94. Friščić, J. et al. The complement system drives local inflammatory tissue priming by metabolic reprogramming of synovial fibroblasts. Immunity $\mathbf{5 4}$ 1002-1021.e10 (2021)

95. Pratt, J. R., Abe, K., Miyazaki, M., Zhou, W. \& Sacks, S. H. In situ localization of C3 Synthesis in experimental acute renal allograft rejection. Am. J. Pathol. 157, 825-831 (2000).

96. Kulkarni, H. S. et al. Intracellular C3 protects human airway epithelial cells from stress-associated cell death. Am. J. Resp. Cell Mol. 60, 144-157 (2019). This study supports the emerging concept of intracellular complement as key driver of healthy cell homeostasis by showing that intracellular C3 induces cell life-saving autophagy in epithelial cells exposed to oxidative stress.

97. Arbore, G. et al. Complement receptor CD46 co-stimulates optimal human CD8+ T cell effector function via fatty acid metabolism. Nat. Commun. 9 4186 (2018).

98. Kolev, M. et al. Complement regulates nutrient influx and metabolic reprogramming during Th 1 cell responses. Immunity 42, 1033-1047 (2015)

99. Arbore, G. et al. T helper 1 immunity requires complement-driven NLRP3 inflammasome activity in $\mathrm{CD}^{+} \mathrm{T}$ cells. Science 352 , aad 1210 (2016).

100. Yan, B. et al. SARS-CoV-2 drives JAK 1/2-dependent local complement hyperactivation. Sci. Immunol. $\mathbf{6}$, eabg0833 (2021)

This is the first study showing that SARS-CoV-2 induces pathological C3 hyperactivation in infected type II pneumocytes and this results in a shift in the balance from C3 homeostatic activity to pathological activity that contributes to COVID-19 pathology.

101. Hajishengallis, G., Reis, E. S., Mastellos, D. C., Ricklin, D. \& Lambris, J.D. Novel mechanisms and functions of complement. Nat. Immunol. 18, 1288-1298 (2017)

102. Hess, C. \& Kemper, C. Complement-mediated regulation of metabolism and basic cellular processes. Immunity 45, 240-254 (2016).

103. Kulasekararaj, A. G. et al. Terminal complement inhibition dampens the inflammation during COVID-19. Brit J. Haematol. 190, e141-e143 (2020).

104. Laurence, J. et al. Anti-complement C5 therapy with eculizumab in three cases of critical COVID-19. Clin. Immunol. 219, 108555 (2020).

105. Mastaglio, S. et al. The first case of COVID-19 treated with the complement C3 inhibitor AMY-101. Clin. Immunol. 215, $108450(2020)$

106. Mastellos, D. C. et al. Complement C3 vs C5 inhibition in severe COVID-19: early clinical findings reveal differential biological efficacy. Clin. Immunol. 220 108598 (2020).

107. Urwyler, P. et al. Treatment of COVID-19 with conestat alfa, a regulator of the complement, contact activation and kallikrein-kinin system. Front. Immunol. 11, 2072 (2020).
108. Zelek, W. M. et al. Complement inhibition with the C5 blocker LFG316 in severe COVID-19. Am. J. Resp. Crit. Care 202, 1304-1308 (2020)

109. Diurno, F. et al. Eculizumab treatment in patients with COVID-19: preliminary results from real life ASL Napoli 2 Nord experience. Eur. Rev. Med. Pharm. 24 4040-4047 (2020).

110. Cavalli, G. et al. Interleukin-1 blockade with high-dose anakinra in patients with COVID-19, acute respiratory distress syndrome, and hyperinflammation: a retrospective cohort study. Lancet Rheumatol. 2 , e325-e331 (2020).

111. Merad, M., Subramanian, A. \& Wang, T. T. An aberrant inflammatory response in severe COVID-19. Cell Host Microbe 29, 1043-1047 (2021).

112. Kyriazopoulou, E. et al. Early treatment of COVID-19 with anakinra guided by soluble urokinase plasminogen receptor plasma levels: a double-blind, randomized controlled phase 3 trial. Nat. Med. 27, 1752-1760 (2021).

113. WHO Rapid Evidence Appraisal for COVID-19 Therapies (REACT) Working Group. Association between administration of IL- 6 antagonists and mortality among patients hospitalized for COVID-19. JAMA 326, 499-518 (2021).

114. RECOVERY Collaborative Group. Dexamethasone in hospitalized patients with Covid-19. N. Engl. J. Med. 384, 693-704 (2020).

This seminal trial shows that anti-inflammatory drug application can ameliorate COVID-19.

115. REMAP-CAP Investigators. Interleukin- 6 receptor antagonists in critically ill patients with Covid-19. N. Engl. J. Med. 384, 1491-1502 (2021).

116. Kalil, A. C. et al. Baricitinib plus remdesivir for hospitalized adults with Covid-19. N. Engl. J. Med. 384, 795-807 (2021)

117. Cao, Y. et al. Ruxolitinib in treatment of severe coronavirus disease 2019 (COVID-19): a multicenter, single-blind, randomized controlled trial. J. Allergy Clin. Immun. 146, 137-146.e3 (2020).

118. Cantini, F. et al. Baricitinib therapy in COVID-19: a pilot study on safety and clinical impact. J. Infect. $\mathbf{8 1}$ 318-356 (2020)

119. Bronte, V. et al. Baricitinib restrains the immune dysregulation in severe COVID-19 patients. J. Clin. Invest. 130, 6409-6416 (2020).

120. Annane, D. et al. Eculizumab as an emergency treatment for adult patients with severe COVID-19 in the intensive care unit: a proof-of-concept study. Eclinicalmedicine 28, 100590 (2020).

121. Declercq, J. et al. Zilucoplan in patients with acute hypoxic respiratory failure due to COVID-19 (ZILU-COV): A structured summary of a study protocol for a randomised controlled trial. Trials 21, 934 (2020).

122. Vlaar, A. P. J. et al. Anti-C5a antibody IFX-1 (vilobelimab) treatment versus best supportive care for patients with severe COVID-19 (PANAMO): an exploratory, open-label, phase 2 randomised controlled trial. Lancet Rheumatol. 2, e764-e773 (2020).

123. FDA. COVID-19 vaccines. https://www.fda.gov/ emergency-preparedness-and-response/coronavirusdisease-2019-covid-19/covid-19-vaccines (2021)

124. EMA. COVID-19 vaccines. https://www.ema.europa.eu/ en/human-regulatory/overview/public-health-threats/ coronavirus-disease-covid-19/treatments-vaccines/ vaccines-covid-19/covid-19-vaccines-authorised (2021).

125. Bloomberg. Bloomberg vaccine tracker. https://www. bloomberg.com/graphics/covid-vaccine-tracker-globaldistribution/\#global (2021).

126. Greinacher, A. et al. Thrombotic thrombocytopenia after ChAdOx $1 \mathrm{nCov}-19$ vaccination. N. Engl. J. Med. 384, 2092-2101 (2021)

127. Choi, P. Y.-I. Thrombotic thrombocytopenia after ChAdOx 1 nCoV-19 vaccination. N. Engl. J. Med. 385 e11 (2021).

128. Muir, K.-L., Kallam, A., Koepsell, S. A. \& Gundabolu, K Thrombotic thrombocytopenia after Ad26.COV2.S vaccination. N. Engl. J. Med. 384, 1964-1965 (2021).

129. Sadoff, J., Davis, K. \& Douoguih, M. Thrombotic thrombocytopenia after Ad26.COV2.S vaccination response from the manufacturer. N. Engl. J. Med. 384, 1965-1966 (2021)

130. See, I. et al. US case reports of cerebral venous sinus thrombosis with thrombocytopenia after Ad26.COV2.S vaccination, March 2 to April 21, 2021. JAMA 325, 2448-2456 (2021).
131. Graf, T. et al. Immediate high-dose intravenous immunoglobulins followed by direct thrombin-inhibitor treatment is crucial for survival in Sars-Covid-19. adenoviral vector vaccine-induced immune thrombotic thrombocytopenia VITT with cerebral sinus venous and portal vein thrombosis. J. Neurol. 268, 4483-4485 (2021).

132. Pomara, C. et al. Post-mortem findings in vaccineinduced thrombotic thombocytopenia. Haematologica 106, 2291-2293 (2021).

133. Hill, A., DeZern, A. E., Kinoshita, T. \& Brodsky, R. A Paroxysmal nocturnal haemoglobinuria. Nat. Rev. Dis. Prim. 3, 17028 (2017)

134. Scheuerle, A. F., Serbecic, N. \& Beutelspacher, S. C. Paroxysmal nocturnal hemoglobinuria may cause retinal vascular occlusions. Int. Ophthalmol. 29, 187-190 (2009).

135. Tiede, A et al. Prothrombotic immune thrombocytopenia after COVID-19 vaccine. Blood 138, 350-353 (2021)

136. CDC COVID-19 Response Team. Preliminary estimates of the prevalence of selected underlying health conditions among patients with coronavirus disease 2019 - United States, February 12-March 28, 2020. MMWR Morb. Mortal. Wkly Rep. 69 382-386 (2020).

137. Jordan, R. E., Adab, P. \& Cheng, K. K. Covid-19: risk factors for severe disease and death. $B M J 368$, m1198 (2020).

138. Wenzel, U. O., Kemper, C. \& Bode, M. The role of complement in arterial hypertension and hypertensive end organ damage. Br. J. Pharmacol. 178, 2849-2862 (2021).

139. Lappegård, K. T. et al. A vital role for complement in heart disease. Mol. Immunol. 61, 126-134 (2014).

140. Arason, G. J. et al. Smoking and a complement gene polymorphism interact in promoting cardiovascular disease morbidity and mortality. Clin. Exp. Immunol. 149, 132-138 (2007)

141. Copenhaver, M., Yu, C.-Y. \& Hoffman, R. P. Complement components, $\mathrm{C} 3$ and $\mathrm{C} 4$, and the metabolic syndrome. Curr. Diabetes Rev. 15, 44-48 (2018)

142. Goronzy, J. J. \& Weyand, C. M. Understanding immunosenescence to improve responses to vaccines. Nat. Immunol. 14, 428-436 (2013).

143. Pietrobon, A. J., Teixeira, F. M. E. \& Sato, M. N. Immunosenescence and inflammaging: risk factors of severe COVID-19 in older people. Front. Immunol. 11, 579220 (2020).

144. da Costa, M. G. et al. Age and sex-associated changes of complement activity and complement levels in a healthy Caucasian population. Front. Immunol. 9 2664 (2018).

145. Cardoso, A. L. et al. Towards frailty biomarkers candidates from genes and pathways regulated in aging and age-related diseases. Ageing Res. Rev. 47, 214-277 (2018)

146. Shi, Q. et al. Complement C3 deficiency protects against neurodegeneration in aged plaque-rich APP/PS1 mice. Sci. Transl. Med. 9, eaaf6295 (2017).

147. Naito, A. T. et al. Complement C1q activates canonical Wnt signaling and promotes aging-related phenotypes. Cell 149, 1298-1313 (2012).

\section{Acknowledgements}

Research described in this article was supported (in part) by the Intramural Research Program of the US National Institute of Diabetes and Digestive and Kidney Diseases (project number ZIA/DK075149 (B.A).) and the US National Heart, Lung, and Blood Institute (project number ZIA/HI006223 (C.K.)), by an FWO COVID-19 grant to B.N.L., and by a COVID-19 grant from the Fondazione Italcementi Cav. Lav. CARLO PESENTI to M.N.

\section{Author contributions}

The authors contributed equally to all aspects of the article.

\section{Competing interests}

The authors declare no competing interests.

\section{Peer review information}

Nature Reviews Immunology thanks M. Bosmann and the other, anonymous, reviewer(s) for their contribution to the peer review of this work.

\section{Publisher's note}

Springer Nature remains neutral with regard to jurisdictional claims in published maps and institutional affiliations.

C) Springer Nature Limited 2021 